\title{
Desigualdades en seguridad vial por fallecimientos en peatones ${ }^{*}$
}

\author{
Inequalities in Road Safety due to Deaths in Pedestrians
}

\section{Desigualdades em seguurança viária por óbitos em pedestres}

Recibido: 25 de abril de 2019

Revisado: 20 de mayo de 2019

Aceptado: 11 de junio de 2019

\author{
Antonio Ramón Gómez García \\ Universidad Espíritu Santo, Ecuador \\ Zoila Isabel Olivo Cando ${ }^{* * *}$ \\ Universidad Internacional SEK, Ecuador \\ Yolis Yajaira Campos Villalta*** \\ Universidad Internacional SEK, Ecuador \\ Pablo Roberto Suasnavas Bermúde* **** \\ Universidad Internacional SEK, Ecuador \\ Isabel Cárdenas Moncayo ${ }^{* * * * *}$ \\ Universidad de Especialidades Espíritu Santo, Ecuador
}

Cómo citar este artículo: Gómez García, A. R., Olivo Cando, Z., Campos Villalta, Y., Suasnavas Bermúdez, P. R. \& Cárdenas Moncayo, I. Desigualdades en seguridad vial por fallecimientos en peatones. Signos. Investigación en Sistemas de Gestión, 12(1), 53-64. DOI: https://doi.org/10.15332/24631140.5420

* $\quad$ Artículo de resultado de investigación.

** Ph.D. Prevención de Riesgos Laborales. Profesor investigador. Facultad de Postgrados, Universidad Espíritu Santo, Ecuador. Correo electrónico: agomezg@uees.edu.ec. Orcid: https://orcid.org/0000-0003-1015-1753.

*** Magíster en Salud y Seguridad Ocupacional de la Universidad Internacional SEK, Ecuador. Correo electrónico: iolivoc@yahoo.com. Orcid: https:// orcid.org/0000-0001-8280-4608.

**** Ph.D. Ciencias Médicas. Profesora Titular de la Facultad de Ciencias del Trabajo y Comportamiento Humano de la Universidad Internacional SEK, Ecuador. Correo electrónico: yolis.campos@uisek.edu.ec. Orcid: https://orcid.org/0000-0002-9874-9049

***** Doctorando en Ciencias Sociales y Jurídicas de la Universidad Rey Juan Carlos, España. Profesor Titular Agregado de la Facultad de Ciencias del Trabajo y del Comportamiento Humano, Universidad Internacional SEK, Ecuador. Correo electrónico: pablo.suasnavas@uisek.edu.ec Orcid: https://orcid.org/0000-0003-3197-760X

***** Doctoranda en Investigación en Psicología. Profesora titular de la Universidad de Especialidades Espíritu Santo. Correo electrónico: isacardenasm@ yahoo.com. Orcid: https://orcid.org/0000-0002-1496-7154. 


\section{RESUMEN}

Los peatones son los usuarios más vulnerables de vialidad, factores como edad y sexo son importantes. El objetivo de este artículo es conocer las desigualdades de mortalidad por atropellos en AT según edad y sexo en Ecuador. Como metodología se realizó un estudio observacional y descriptivo, con datos tomados de la Agencia Nacional de Tránsito. Se calcula DF, TM, AVPP y RR. Como resultado se encontró lo siguiente: 4127 siniestros, 3211 fallecidos por AT a nivel nacional, $19 \%$ peatones, $74 \%$ hombres (relación 3:1 respecto a mujeres); $41 \%$ por conductor del vehículo no ceder paso a peatón y $59 \%$ por imprudencias del peatón; diciembre $(\mathrm{n}=54)$ y mayo $(n=44) 2017$ fueron los meses con mayor mortalidad en área urbana. Ligera disminución de víctimas mortales $\left(\mathrm{y}=-0,909-\mathrm{x}=+42,03 ; \mathrm{R}^{2}=0,282\right) ; \mathrm{TM}$ de 35,3 , hombres $\geq 80$ años con tasas ajustadas más altas, $\geq 65$ años mayor riesgo de fallecimiento, especialmente mujeres, 19.362 AVPP; niñas-mujeres hasta 14 años > IAVPP. Peatones $\geq 65$ años usuarios más vulnerables y de mayor riesgo. En conclusión, las políticas públicas tomadas para reducir accidentabilidad y mortalidad en Ecuador proporcionan resultados de bajo impacto, esto lo confirma el segundo lugar que ocupa el Ecuador en el ranking de mortalidad por AT, de países latinoamericanos analizados en este estudio.

Palabras clave: peatón, desigualdad, mortalidad, usuarios vulnerables de la carretera.

\section{ABSTRACT}

Pedestrians are the most vulnerable road users, factors such as age and sex are important. Objective: To know the inequalities of mortality due to accidents in Traffic Accidents (TA) according to age and sex in Ecuador. Methodology: Observational and descriptive study, data taken from the National Transit Agency. DF, mortality rate (MR), potential years of life lost (PYLL) and relative risk (RR) are calculated. Results: 41,427 accidents, 3,211 deaths due to nationwide TA, $19 \%$ pedestrians, $74 \%$ men (3:1 ratio in comparison with women); $41 \%$ due to vehicle driver not giving way to pedestrians and $59 \%$ due to pedestrian carelessness; December $(\mathrm{n}=54)$ and May $(\mathrm{n}=44) 2017$ were the months with the highest mortality in urban areas. Low decrease in fatalities $\left(y=-0.909-x=+42.03 ; R^{2}=0.282\right) ; M R$ of 35.3 , men $\geq 80$ years with higher adjusted rates, $\geq 65$ years higher risk of death, especially women, 19,362 PYLL; girlswomen up to 14 years old > PYLL Index. Pedestrians $\geq 65$ years more vulnerable and higher risk users. In conclusion, the public policies taken to reduce accident rate and mortality in Ecuador provide low impact results, this is confirmed by the 2nd place that Ecuador occupies in the TA mortality ranking of Latin American countries analyzed in this study.

Keywords: pedestrian, inequality, mortality, vulnerable road users.

\section{RESUMO}

Os pedestres são os usuários mais vulneráveis da viação, sendo muito importantes os fatores como idade e sexo. Objetivo: conhecer as desigualdades de mortalidade por atropelamentos em acidentes de trânsitos (AT) conforme a idade e o sexo no Equador. Metodologia: Estudo observacional e descritivo, dados obtidos da Agência Nacional de Trânsito. Calcula-se DF, TM, AVPP e RR. Resultados: 41.427 acidentes, 3.211 óbitos por AT em nível nacional, $19 \%$ pedestres, $74 \%$ homens (relação 3:1 em relação a mulheres); $41 \%$ por motorista da viatura não ceder o passo a pedestre e $59 \%$ por imprudência de pedestres; dezembro $(n=54)$ e maio $(n=44) 2017$ foram os meses com maior mortalidade em área urbana. 
Leve diminuição de vítimas mortais ( $\mathrm{y}=-0,909$ $\left.\mathrm{x}=+42,03 ; \mathrm{R}^{2}=0,282\right) ; \mathrm{TM}$ de 35,3 , homens $\geq 80$ anos com índices ajustados mais altos, $\geq 65$ anos maior risco de óbito, de maneira especial mulheres, 19.362 AVPP; meninas-mulheres até 14 anos > IAVPP. Pedestres $\geq 65$ anos usuários mais vulneráveis e de maior risco. Conclusões: Políticas públicas adotadas para reduzir acidentalidade e mortalidade no Equador providenciam resultados de baixo impacto, isso confirme $02^{\circ}$ posto ocupado pelo Equador na classificação de mortalidade por AT, de países latino-americanos analisados neste estudo.

Palavras-chave: pedestre, desigualdade, mortalidade, usuários vulneráveis de estradas.

\section{INTRODUCCIÓN}

Los accidentes de tránsito causan aproximadamente 1,2 millones de muertes anualmente, siendo los países de ingresos económicos bajos y medianos los de mayor tasa de mortalidad a escala mundial (Organización Panamericana de la Salud, 2013). Para el 2020 se prevé que, si no se adoptan medidas, la cifra de personas fallecidas podría aumentar a 1,9 millones (OMS, 2015).

La seguridad vial es un tema relativamente reciente en las agendas públicas de Ecuador. A pesar de la tendencia a la disminución de las tasas de mortalidad por accidentes de tránsito (Algora, Russo, Suasnavas, Merino \& Gómez, 2017), los peatones constituyen el grupo de usuarios de la red viaria más vulnerables: en 2016 representaron el 29\% de total de víctimas mortales, principalmente en personas de la cuarta edad de la vida (Algora, Russo, Suasnavas, Merino \& Gómez, 2017b; Peñafiel et al., 2018).
La edad es un factor importante por considerar en el análisis de los atropellos por accidentes de tránsito y difieren de forma considerable por sexo, lo que subraya la necesidad de una recopilación y análisis de datos que permitan un conocimiento actual del problema (OMS, 2013; Onieva et al., 2016).

Esta investigación tuvo como objetivo conocer las desigualdades de mortalidad por atropellos en accidente de tránsito según sexo y grupos de edad en Ecuador. Los resultados hallados en este estudio permiten, por primera vez, generar información sobre esta problemática en salud pública para este tipo de usuarios de la vía, con el fin de concienciar y apoyar en el desarrollo de planes de acción en el país, acordes al Decenio de Acción para la Seguridad Vial 2011-2020 (OMS, 2011).

\section{METODOLOGÍA}

Estudio observacional y descriptivo de 601 casos de víctimas mortales en atropellos por accidentes de tránsito en la población ecuatoriana entre el $1^{\circ}$ de enero de 2017 al 30 junio de 2018, según datos oficiales registrados por la Agencia Nacional de Tránsito (ANT, 2018) y censo realizado en Ecuador (INEC, 2014), permitiendo conocer las desigualdades por atropellos en AT.

Se consideraron como variables de análisis y agrupación el sexo (hombre y mujer), grupos de edad (niños $\leq 14$ años; jóvenes 15 a 24 años; adultos 25 a 64 años y $\geq 65$ años) y distribución por componente temporal según semestres anuales del período de estudio.

Por variables se calcularon la distribución de frecuencias absolutas y relativas, tasas de mortalidad crudas (x 1000000 habitantes) y ajustadas (x 100000 
habitantes), estimación e índice de años de vida potenciales perdidos (AVPP-IAVPP x 10000 habitantes) y riesgo relativo (RR-IC 95\%), empleando la proyección de la población ecuatoriana (17 023408 habitantes) y la esperanza de vida (74,5 en hombres y 80,1 en mujeres) del Instituto Nacional de Estadística y Censos (INEC, 2014).

Estos indicadores de salud ofrecen una visión de la mortalidad innecesariamente prematura y sanitariamente evitable en atropellos por accidente de tránsito en subgrupos vulnerables de la población, además permiten identificar desigualdades de género en seguridad vial (Rodríguez \& Campuzano, 2010).

El procesamiento y análisis de los datos se realizó mediante el paquete Statistic Package for Social Sciences versión 25.0 (SPSS®) y Microsoft Excel 2016®, este último para la representación de figuras.

\section{RESULTADOS Y DISCUSIÓN}

En el período de estudio se han registrado un total de 41427 siniestros, 31593 víctimas con lesión y 3211 personas fallecidas por accidentes de tránsito a nivel nacional. Del total de personas fallecidas, el $19 \%(\mathrm{n}=601)$ corresponden a víctimas mortales por atropellos, de los cuales el $74 \%(n=446)$ son hombres, con una relación 3:1 respecto a las mujeres.

Entre las principales causas de los atropellos con víctimas mortales el $41 \%$ se debe a que el conductor del vehículo a motor no cedió el derecho de vía o preferencia de paso al peatón y el $59 \%$ a imprudencias del mismo peatón: el 49 \% por no transitar por las aceras o zonas de seguridad destinadas para el efecto, $7 \%$ por cruzar la calzada sin respetar la señalización existente (semáforos) y el $3 \%$ por transitar bajo influencia de alcohol, sustancias estupefacientes o psicotrópicas y/o medicamentos. Este hecho ocurre con mayor presencia en las zonas urbanas de las principales provincias del país (no se dispone de información estadística desglosada según distribución geográfica de la ocurrencia del siniestro).

Por número de casos según distribución por componente temporal, los meses donde notablemente se produjeron más víctimas mortales en atropellos fueron diciembre $(\mathrm{n}=54)$ y mayo $(\mathrm{n}=44)$ de 2017 seguidos de marzo, julio y agosto del mismo año. Se observa una ligera tendencia a la disminución de las víctimas mortales $(\mathrm{y}=-0,909-\mathrm{x}$ $\left.=+42,03 ; \mathrm{R}^{2}=0,282\right)$, en particular, del primer semestre de 2017 a 2018 ha disminuido en un $-37 \%$ el número de casos: 225 (promedio $=38 \pm 4$ ) casos en el primer semestre y 235 (promedio $=39 \pm 8$ ) en el segundo semestre de 2017; y primer semestre de 2018 con 141 casos $($ promedio $=24 \pm 4)($ figura 1$)$.

Por frecuencia de casos según grupo etario (tabla 1), predominan las personas adultas entre los 25 a 64 años $(\mathrm{n}=284 ; 47 \%)$ y los mayores a 65 años o más $(\mathrm{n}=162$; $27 \%$ ) con respecto al total de fallecidos en atropellos por accidente de tránsito.

La tasa de mortalidad cruda por atropellos en el período de estudio fue de 35,3 muertes por cada 1000000 habitantes en el total de la población. Con mayor medida en hombres y mujeres mayores a 65 años o más, con tasas de mortalidad ajustada por cada 100000 habitantes de 18,1 y 9,1, respectivamente. Las personas de 80 años 0 más presentan mayor número de casos y las tasas de mortalidad ajustadas más altas: en hombres de 41,2 y mujeres de 20,2 por cada 100000 habitantes. 
Figura 1. Distribución semestral de fallecimientos por atropellos según sexo: 2017-2018

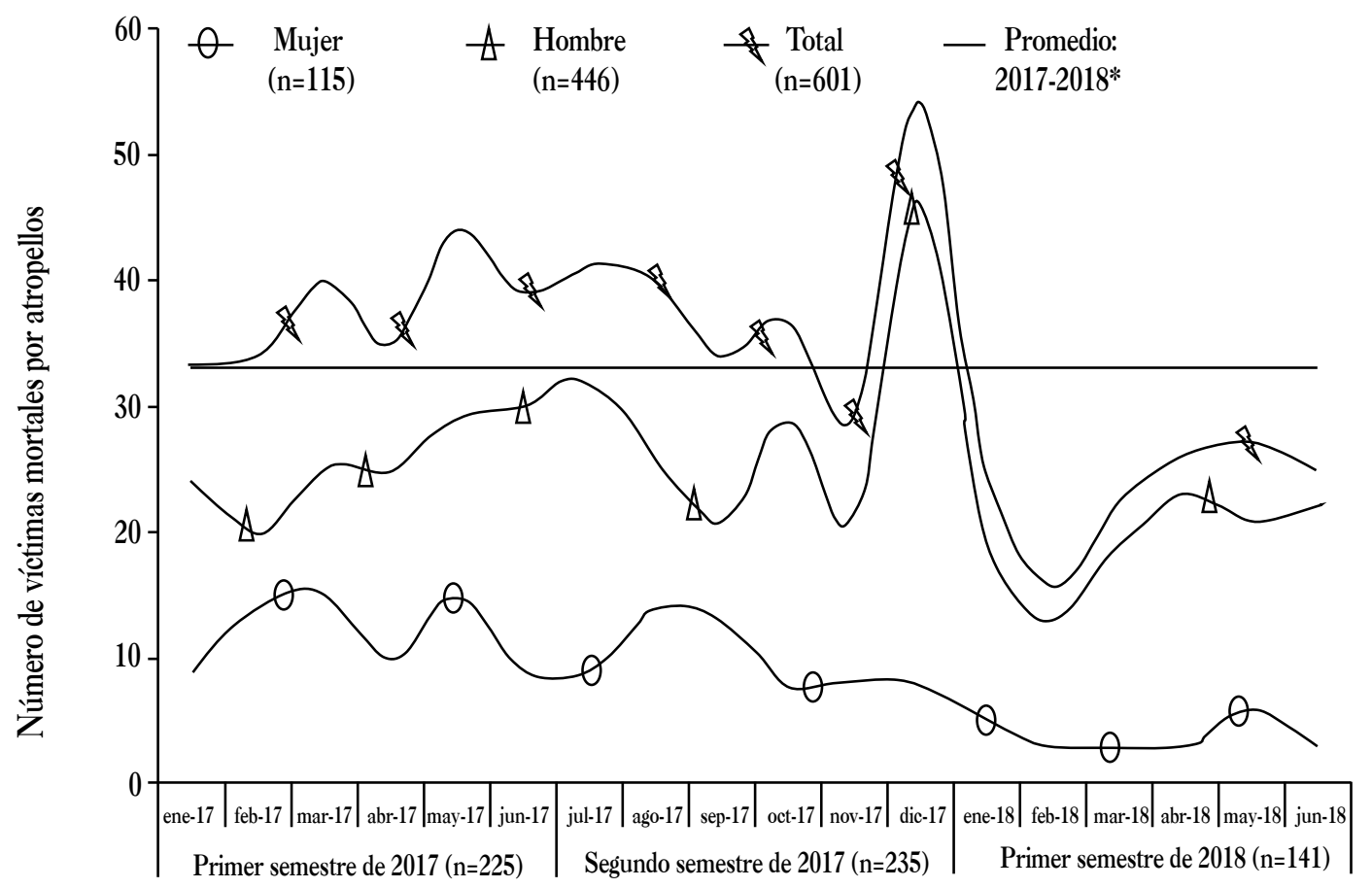

Fuente: elaboración propia.

Tabla 1. Tasas de mortalidad por accidentes de tránsito según grupos etarios y sexo: 17-18

\begin{tabular}{|c|c|c|c|c|c|c|}
\hline \multirow[t]{2}{*}{ Grupos / Edad } & \multicolumn{3}{|c|}{ Mujer } & \multicolumn{3}{|c|}{ Hombre } \\
\hline & $\mathrm{n}(\% \mathrm{n})$ & T.M. ${ }^{a}$ & T.M. $^{\mathrm{b}}$ & $\mathrm{n}(\% \mathrm{n})$ & T.M. ${ }^{a}$ & T.M. ${ }^{b}$ \\
\hline Niños & $30(19,4)$ & 6,0 & 1,2 & $60(13,5)$ & 12,0 & 2,3 \\
\hline$\leq 4$ & $14(9,0)$ & 8,4 & 1,7 & $35(7,8)$ & 21,0 & 4,1 \\
\hline $5-9$ & $11(7,1)$ & 6,5 & 1,3 & $15(3,4)$ & 8,9 & 1,7 \\
\hline $10-14$ & $5(3,2)$ & 3,0 & 0,6 & $10(2,2)$ & 6,0 & 1,2 \\
\hline Jóvenes & $14(9,0)$ & 4,6 & 0,9 & $51(11,4)$ & 16,6 & 3,3 \\
\hline $15-19$ & $10(6,5)$ & 6,3 & 1,3 & $20(4,5)$ & 12,6 & 2,5 \\
\hline $20-24$ & $4(2,6)$ & 2,7 & 0,5 & $31(7,0)$ & 21,0 & 4,2 \\
\hline Adultos & $51(32,9)$ & 4,6 & 0,9 & $233(52,2)$ & 30,2 & 6,2 \\
\hline $25-29$ & $7(4,5)$ & 5,2 & 1,0 & $43(9,6)$ & 31,7 & 6,4 \\
\hline $30-34$ & $7(4,5)$ & 5,6 & 1,1 & $27(6,1)$ & 21,6 & 4,4 \\
\hline $35-39$ & $3(1,9)$ & 2,6 & 0,5 & $26(5,8)$ & 22,6 & 4,7 \\
\hline
\end{tabular}


Antonio Ramón Gómez García, Zoila Isabel Olivo Cando, Yolis Yajaira Campos Villalta, Pablo Roberto Suasnavas Bermúdez, Isabel Cárdenas Moncayo

\begin{tabular}{|c|c|c|c|c|c|c|}
\hline \multirow{2}{*}{ Grupos / Edad } & \multicolumn{5}{|c|}{ Mujer } & \multicolumn{3}{c|}{ Hombre } \\
\hline & $\mathrm{n}(\% \mathrm{n})$ & T.M. ${ }^{\mathrm{a}}$ & T.M. $^{\mathrm{b}}$ & $\mathrm{n}(\% \mathrm{n})$ & ${\text { T.M. }{ }^{\mathrm{a}}}^{\text {T.M. }^{\mathrm{b}}}$ \\
\hline $40-44$ & $2(1,3)$ & 1,9 & 0,4 & $28(6,3)$ & 27,2 & 5,7 \\
\hline $45-49$ & $6(3,9)$ & 6,6 & 1,3 & $34(7,6)$ & 37,5 & 7,9 \\
\hline $50-54$ & $6(3,9)$ & 7,5 & 1,4 & $21(4,7)$ & 26,4 & 5,5 \\
\hline $55-59$ & $10(6,5)$ & 14,7 & 2,8 & $24(5,4)$ & 35,3 & 7,4 \\
\hline $60-64$ & $10(6,5)$ & 18,0 & 3,4 & $30(6,7)$ & 54,1 & 11,4 \\
\hline Mayores & $60(38,7)$ & 49,1 & 9,1 & $102(22,9)$ & 83,5 & 18,1 \\
\hline $65-69$ & $9(1,0)$ & 20,8 & 3,9 & $16(3,6)$ & 37,0 & 7,8 \\
\hline $70-74$ & $13(8,4)$ & 40,3 & 7,5 & $24(5,4)$ & 74,5 & 16,0 \\
\hline $75-79$ & $11(7,1)$ & 49,1 & 9,0 & $17(3,8)$ & 75,9 & 16,7 \\
\hline$\geq 80$ & $27(17,4)$ & 111,2 & 20,2 & $45(10,1)$ & 185,3 & 41,2 \\
\hline
\end{tabular}

Fuente: elaboración propia.

Nota: a Tasa de mortalidad cruda x 1000000 habitantes. b Tasa de mortalidad ajustada x 100000 habitantes.

En cuanto a los AVPP en atropellos por accidentes de tránsito, este fue de 19362 años de vida perdidos (tabla 2), siendo los grupos de edad de mayor pérdida los niños (6243) y los adultos (8806), mayormente hombres. Asimismo, el IAVPP para el período de estudio sitúa a las niñas-mujeres hasta los 14 años con las de mayor pérdida por esta causa dentro de su grupo de edad (8,9 10000 habitantes). Igualmente, se observa en hombres hasta los 65 años de edad valores similares de pérdida en años, superando a las mujeres, a excepción de los 65 años de edad o más (3,6 x 10000 habitantes).

Finalmente, las mujeres $(\mathrm{RR}=5,07 ; \mathrm{IC} 95 \%=4,17$ $6,18)$ y los hombres (RR $=3,41 ; \mathrm{IC} 95 \%=2,89-4,06)$ de 65 años o más presentan mayor riesgo de fallecimiento en atropellos por accidente de tránsito que el resto de la población ecuatoriana (figura 2).
Tabla 2. Años de vida perdidos por accidentes de tránsito según sexo y grupos de edad

\begin{tabular}{|c|c|c|c|c|}
\hline $\begin{array}{c}\text { Grupos/ } \\
\text { Edad }\end{array}$ & \multicolumn{2}{|c|}{ Mujer } & \multicolumn{2}{c|}{ Hombre } \\
\hline & AVPP & IAVPP & AVPP & IAVPP \\
\hline Niños & 2.193 & 8,9 & 4.050 & 15,8 \\
\hline Jóvenes & 848 & 5,6 & 2.805 & 18 \\
\hline Adultos & 1.816 & 4,6 & 6.990 & 18,7 \\
\hline Mayores & 456 & 6,9 & 204 & 3,6 \\
\hline
\end{tabular}

Fuente: elaboración propia.

Nota: AVPP = años de vida potenciales perdidos $\times 10000$ habitantes. IAVPP índice de años de vida potenciales perdidos x 10000 habitantes. 
Figura 2. Riesgo relativo (IC95\%) de fallecimiento por accidentes de tránsito según sexo y grupos de edad: 2017-2018
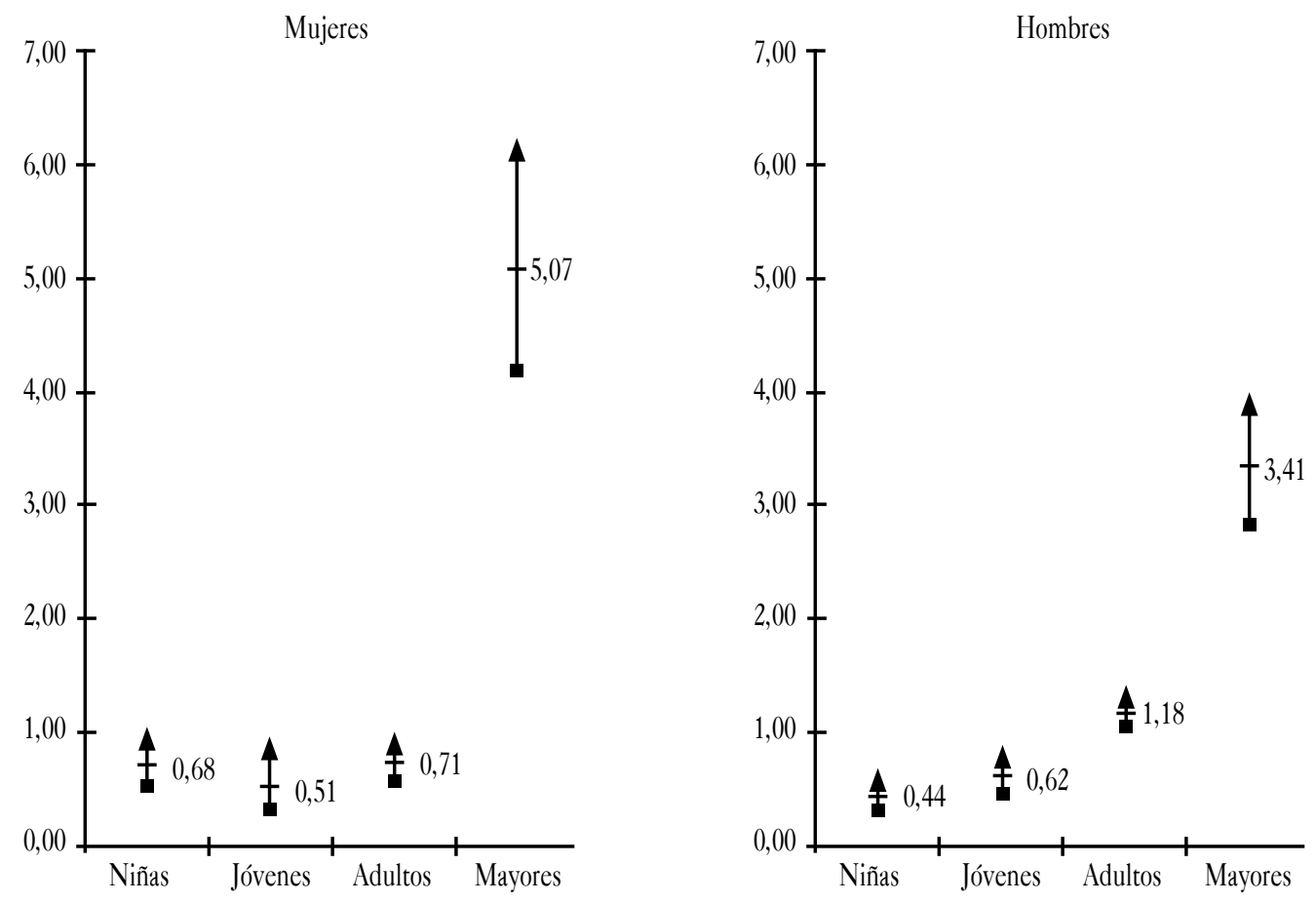

Fuente: elaboración propia.

A diferencia de los países de ingresos económicos altos, la población ecuatoriana se caracteriza por ser joven y su esperanza de vida sigue creciendo (INEC, 2014). Pese a que las personas mayores a 65 años o más representan solo un $7 \%$ de la población, este grupo supone el $23 \%$ de las víctimas mortales por atropellos en accidentes de tránsito en el período de estudio.

Los hombres de 25 años o más presentan proporciones y tasas de mortalidad notablemente elevadas respecto a las mujeres, similar a lo observado en países de ingresos bajos y medianos (OPS, 2013; Zangooei, Shafahi \& Zangooei, 2013). En particular, los peatones de 65 años o más muestran mayor riesgo de fallecimiento por esta causa (Sadeghi, Samadirad \& Moslemi, 2018) debido a los traumatismos provocados, especialmente en mujeres de esta edad (Ribeiro, Partezani, Dos Santos \& Bussi, 2016; Damsere, Palk \& King, 2017).
Los diferentes modos de exposición, situación socioeconómica y nivel educativo de la población afectada podrían ayudar a interpretar las desigualdades en seguridad vial por sexo y grupos de edad en los atropellos por accidentes de tránsito (Dandona, Kumar, Ameer, Ahmed \& Dandona, 2008).

Los años de vida potenciales perdidos por esta causa afectan principalmente a niños, jóvenes y adultos de ambos sexos, por lo tanto, deben considerarse como una población de alto riesgo por el impacto social y económico negativo en términos de productividad para el país en el momento de planificar programas preventivos y políticas públicas (Vanegas \& Cárdenas, 2011).

En cuanto a la distribución por componente temporal, como era de esperarse, los meses con mayor número de casos en atropellos por accidentes de tránsito 
corresponden a períodos vacacionales por el incremento de la movilidad peatonal. La leve tendencia a la disminución apreciada por semestres puede enmascarar la realidad, los accidentes de tránsito siguen siendo un problema de gran preocupación social.

Los procesos psicológicos y comportamentales juegan un papel esencial. En este sentido, se diferencian los errores, como no mirar antes de cruzar la calle $(46,5 \%)$ o cruzarla hablando por teléfono $(32,2 \%)$ e infracciones por incumplimiento de las normas de tránsito tanto de conductores como de peatones (Buedo, Silberman \& Stickar, 2016). Además, la pérdida de motricidad, visión y audición en la población mayor a 60 años puede contribuir como factores importantes en la probabilidad de los atropellos por accidente de tránsito (Onishi et al., 2005). En nuestro estudio se revela que las imprudencias de ambos usuarios de la vía son las principales causas de los fallecimientos por este flagelo en el país (Gómez, Ayala \& Campos, 2018).

Aunque son escasos los estudios sobre los factores de accidentalidad en este contexto, una encuesta realizada a 671 personas en la ciudad ecuatoriana de Loja devela que la mayoría de peatones sienten miedo al cruzar las calles, principalmente en menores a 30 años y mayores a 55 años de edad (García, Zárate, Segarra \& González, 2017). No obstante, los peatones de todas las edades subestiman el peligro de ser atropellados y adoptan conductas inseguras (Galanis, Botzoris \& Eliou, 2017).

Ante esta problemática, diferentes investigaciones proponen medidas preventivas relacionadas con el (i) factor humano, (ii) con la propia vía y (iii) con los vehículos para reducir el número de víctimas en atropellos por accidentes de tránsito, según se muestra a continuación.

\section{Medidas relacionadas con el factor humano}

La educación vial es una necesidad social que podría mejorar el conocimiento, concienciación y comportamiento en los usuarios de la vía de tránsito (Fernández, Ávila \& Milanés, 2017), en particular en lo referente a los mecanismos psicológicos que caracterizan a las violaciones o transgresiones, errores y lapsus de peatones y conductores (Moyano, Jucksch \& Bianchi, 2014). No obstante, en un análisis sobre la revisión sistemática de todas aquellas revisiones de la literatura publicadas que analizan la efectividad de las intervenciones para reducir los accidentes de tránsito se evidencia que la educación vial no se encuentra entre las acciones más efectivas si no están acompañadas del apoyo de otro tipo de medidas (Novoa, Pérez \& Borrell, 2009).

\section{Medidas relacionadas con la vía}

En cuanto a las medidas relacionadas para mejorar la infraestructura vial para reducir el riesgo de atropellos a peatones, destacan las siguientes: la iluminación, la señalización, los reductores de velocidad, los pasos elevados, las aceras, pasos de peatones 3D, etc. (OMS, 2013). Establecer límites de velocidad en zonas urbanas es una de las medidas más eficaces y contribuye a mejorar la seguridad de todos los usuarios de la vía, reduciendo el riesgo de presentar daños tanto en conductores, pasajeros y peatones. Un trabajo realizado en Londres demostró que la introducción de límites de velocidad a $32 \mathrm{~km} / \mathrm{h}$ en zonas urbanas redujo el número de víctimas en un 42\% (Grundy, Steinbach, Edwards, Green, Armstrong \& Wilkinson, 2009).

Por ello, sería recomendable evaluar la infraestructura vial destinada a la movilidad peatonal en entornos urbanos de las ciudades del país desde un enfoque morfológico, funcional y ambiental (Valenzuela \& Talavera, 2015). 


\section{Medidas relacionadas con los vehículos}

La importación al país de vehículos que dispongan de sistemas de detección de peatones con frenada autónoma de emergencia (AEB), sistema antibloqueo de frenos $(\mathrm{ABS})$ y control de estabilidad (ESP), entre otros elementos de seguridad activa, podría reducir la probabilidad de los accidentes (Hamdane, Serrea, Masson \& Anderson, 2015).

Los ensayos realizados por el Programa Europeo de Evaluación de Automóviles Nuevos (Euro NCA) demuestra que incorporando estos elementos de seguridad activa en los vehículos podrín prevenir uno de cada cinco atropellos y hasta el $40 \%$ de los fallecimientos. Lastimosamente, la población ecuatoriana no está preparada económicamente para la adquisición de estos vehículos de alta gama por los altos costos que suponen. Ante esta limitación, el mantenimiento preventivo periódico del vehículo es una medida más de seguridad.

Adicionalmente de las medidas preventivas planteadas anteriormente, la asistencia sanitaria oportuna a las personas involucradas en accidentes de tránsito en tiempos razonables puede prevenir el número de fallecimientos, en especial en peatones niños y adultos debido a su fragilidad (Segura, Cardona, Berbesí \& Agudelo, 2017).

Entre las principales limitaciones del estudio destaca la falta de información estadística oficial para realizar estudios causales e identificar geoespacialmente las vías de tránsito donde se han registrado los eventos por atropellos, imposibilitando aumentar el nivel de evidencia (Grundy et al., 2009; Galanis et al., 2017, Cervantes, Trejo, Rodríguez \& Castañeda, 2018). Especialmente, en lo referente a los mecanismos psicológicos y socioculturales que inciden en las conductas imprudentes de los usuarios de la vía por sexo y grupos de edad (Cinnamon, Schurman \& Hameed, 2011).

\section{CONCLUSIONES}

En conclusión, los resultados del presente trabajo permiten por primera vez presentar evidencias sobre las desigualdades en seguridad vial en subgrupos vulnerables de la población ecuatoriana según sexo y edad. Se revela claramente que los peatones de 65 años de edad o más son los usuarios de la vía más vulnerables y de mayor riesgo.

Las personas mayores son, al igual que en niños, grupos prioritarios a los que se deben dirigir las políticas públicas (Onieva et al., 2016), orientadas a concienciar y apoyar el desarrollo de planes de acción en el país (OMS, 2011).

Las políticas públicas tomadas para reducir la accidentabilidad y morbilidad en el Ecuador, a pesar de la rigidez de las leyes están dando resultados de bajo impacto, lo que se demuestra con la ubicación del Ecuador en el ranking de mortalidad por AT $\left(2^{\circ}\right.$ lugar en Latinoamérica), de los países analizados en este estudio (LatinAmérican Post, 2018).

La OMS recomienda el uso de un enfoque sistémico que contemple al usuario, los vehículos y la infraestructura para mejorar la seguridad vial. Este enfoque incluye proporcionar redes viales eficientes y fomentar el uso de medios de transporte más seguros, sustentables y de precios accesibles. No solo requiere una implementación simultánea, sino de una firme voluntad política y una estrecha colaboración con los distintos sectores involucrados (OPS, 2009).

De igual forma, se propone elaborar estrategias nacionales a nivel ministerial donde se confirmen la especificación de responsabilidades y de rendición de cuentas de los organismos competentes con respecto a la ejecución de programas de trabajo básicos; la promoción de iniciativas de gestión de la seguridad vial como la 
nueva norma ISO 39001, relativa a los riesgos identificados y considerando la responsabilidad compartida de los usuarios de la vía de respetar la ley y actuar con responsabilidad; el establecimiento y mantenimiento de los sistemas de recopilación de datos necesarios para proporcionar información de referencia y seguir avances logrados en materia de reducción de las defunciones y los traumatismos causados por el tránsito (Plan Mundial Decenio de Seguridad Vial, 2011-220).

\section{REFERENCIAS}

Algora, A. F., Russo, M., Suasnavas, P. R., Merino, P. \& Gómez, A. R. (2017). Tendencias de los accidentes de tránsito en Ecuador: 2000-2015. Revista Gerencia y Políticas de Salud, 16(33), 52-58. https://doi. org/10.11144/Javeriana.rgps16-33.tate.

Algora, A. F., Russo, M., Suasnavas, P. R., Merino, P. \& Gómez, A. R. (2017). Epidemiological study of fatal road traffic accidents in Ecuador. Australasian Medical Journal, 10(3), 238-245. DOI: https://doi. org/10.21767/AMJ.2017.2951.

Buedo, P., Silberman, P. \& Stickar, A. (2016). Errores humanos autorreferenciados por los peatones del sistema vial de la ciudad de Bahía Blanca, Argentina: estudio descriptivo observacional. Revista Médica del Uruguay, 32(1), 36-42. Recuperado de http://www.scielo.edu.uy/scielo.php?script=sci_ abstract\&pid=S1688-03902016000100005\&lng=e s\&nrm=iso.

Cervantes, A., Trejo, L., Rodríguez, C. \& Castañeda, I. (2018). Vulnerable Road Users in Mexico: The Perils of Pedestrian Mobility in Developing Countries. Juniper Online Journal of Public Health, 3(1),
555-605. Recuperado de https://juniperpublishers. com/jojph/pdf/JOJPH.MS.ID.555605.pdf.

Cinnamon, J., Schurman, N. \& Hameed, S.M. (2011). Pedestrian injury and human behavior: observing road-rule violations at high-incident intersections. PLoS One, 6, e21063.

Damswer, J., Schurman, N. \& Hameed, S. M. (2011). Pedestrian injury and human behavior: observing road-rule violations at high-incident intersections. 6(6), e21063 DOI: https://doi.org/10.1371/journal.pone.0021063.

Damsere, J., Palk, G. \& King, M. (2017). Road accident fatality risks for "vulnerable" versus "protected" road users in northern Ghana. Traffic Injury Prevention, 18(7), 736-743. DOI: https://doi.org/10.1080/1 5389588.2017 .1302083$.

Dandona, R., Kumar, G.A., Ameer, M.A., Ahmed, G. M. \& Dandona L. (2008). Incidence and burden of road traffic injuries in urban India. Injury Prevention, 14, 354-359. DOI: https://doi.org/10.1080/153 89588.2017.1302083.

Ecuador. Agencia Nacional de Tránsito (2018). Estadisticas de transporte terrestre y seguridad vial. Recuperado de http://www.ant.gob.ec/index.php/noticias/ estadisticas\#.V4I6YFcWUvP.

Fernández, J. E., Ávila, A. M. \& Milanés, R. (2017). La educación vial asistida por tecnología 3D: un modelo de su enseñanza-aprendizaje. Universidad y sociedad, 9(2), 130-134. Recuperado de http:// scielo.sld.cu/scielo.php?script=sci_arttext\&pi $d=S 2218-36202017000300020$.

Galanis, A., Botzoris, G. \& Eliou, N. (2017). Pedestrian road safety in relation to urban road type and traffic 
flow. Transportation Research Procedia, 24, 220-222. DOI: https://doi.org/10.1016/j.trpro.2017.05.111.

García Y., Zárate B., Segarra S. \& González J. (2017). Percepción general de la seguridad vial en la ciudad de Loja (Ecuador). Revista Cumbres, 3(2), 9-16. Recuperado de file:///C:/Users/ADMIN/Downloads/ DialnetPercepcionDeLaSeguridadVialEnLaCiudadDeLojaEcuador-6550762.pdf.

Gómez, A. R., Ayala, M. \& Campos, Y. Y. (2018). Caracterización de 1967 casos de fallecimientos por accidentes de tránsito en Ecuador. Revista Espirales, 2(21), 57-68. Recuperado de http://www.revistaespirales.com/index.php/es/article/view/342.

Grundy, C., Steinbach, R., Edwards, P., Green, J., Armstrong, B. \& Wilkinson, P. (2009). Effect of $20 \mathrm{mph}$ traffic speed zones on road injuries in London, 19862006: controlled interrupted time series analysis. BMJ. 339. DOI: https://doi.org/10.1136/bmj.b4469.

Gutiérrez, J. (2018). Accidentes Viales en América Latina. Latinamerican post. Recuperado de https://latinamericanpost.com/es/18978-accidentes-viales-enamerica-latina.

Hamdane, H., Serrea, T., Masson, C. \& Anderson, R. (2015). Issues and challenges for pedestrian active safety systems based on real world accidents. Accident Analysis \& Prevention, 82, 53-60. DOI: https:// doi.org/10.1016/j.aap.2015.05.014.

Instituto Nacional de Estadística y Censos. (2014). Compendio estadístico 2014. Recuperado de: http:// www.ecuadorencifras.gob.ec/compendio-estadistico-2014/
Lloyd's Register (2018). ISO 39001. Sistema de Gestión de la SeguridadVial. Recuperado de https://www.lr.org/ es-cl/iso-39001/

Moyano, E., Jucksch, R. \& Bianchi, A. (2014). Aportaciones a las ciencias de la salud: el comportamiento peatonal arriesgado de chilenos y brasileños. Terapia Psicológica, 32(3), 227-234. DOI: http://dx.doi. org/10.4067/S0718-48082014000300005.

Oniev, A. M., Pérez, K. \& Borrell, C. (2009). Efectividad de las intervenciones de seguridad vial basadas en la evidencia: una revisión de la literatura. Gaceta Sanitaria, 23(6), 553.e1-553.e14. Recuperado de http:// scielo.isciii.es/pdf/gs/v23n6/revision.pdf.

Onieva, M.A., Martínez, V., Lardelli, P., Jiménez Moleón, J. J., Amezcua, C., Luna del Castillo, J. D. \& Jiménez Mejías, E. (2016). Gender and age differences in components of traffic-related pedestrian death rates: exposure, risk of crash and fatality rate. Injury Epidemiology, 3(14), 2-10. Recuperado de file://C:/Users/gutie/Downloads/ gender-and-age-differences-in-components-oftraffic-related-pedestrian-death-rates-exposurerisk-of-crash-and-fatality-rate.pdf.

Onishi, J., Masuda, Y., Suzuki, Y., Gotoh, T., Kawamura, T. \& Iguchi, A. (2005). The pleasurable recreational activities among community-dwelling older adults. A rchives of Gerontology and Geriatrics, 43(2), 147-155. DOI https://doi.org/10.1016/j.archger.2005.10.003.

Organización Mundial de la Salud. (2015). Global status report on road safety 2015. Recuperado de http://apps.who.int/iris/bitstream/ 10665/189242/1/9789241565066_eng.pdf?ua=1. 
Organización Mundial de la Salud. (2013). Seguridad peatonal: manual de seguridad vial para instancias decisorias y profesionales. Recuperado de http://apps.who.int/iris/bitstream/han dle/10665/128043/9789243505350_spa.pdf;jsessio nid=4E1CC90DD889D7D326853FE661568F42? sequence $=1$ ).

Organización Mundial de la Salud. (2011). Plan Mundial para el Decenio de Acción para la Seguridad Vial 2011-2020. Recuperado de http://www.who.int/roadsafety/decade_of_action/plan/plan_spanish.pdf.

Organización Panamericana de la Salud. (2013). Status report on road safety in Americas region. Recuperado de http://www.paho.org/hq/index.php?option=com_docman\&task=doc_ view\&gid=20941\&Itemid=270.

Organización Panamericana de la Salud. (2009). Informe sobre el Estado de la Seguridad Vial en la Región de las Américas. Washington, D. C.: ISBN: 978-92-7533069-2.

Peñafiel, A. P., Portalanza, A., Espinoza, C. E., Merino, P. \& Gómez, A. R. (2018). Mortalidad y años de vida potencialmente perdidos por accidentes de tránsito en Ecuador. Revista CienciAmérica, 7(1), 11-21. Recuperado de file:///C:/Users/gutie/Downloads/ DialnetMortalidadYAnosDeVidaPotencialmentePerdidosPorAcci-6250912.pdf.

Ribeiro, A., Partezani, R.A., Dos Santos, C.B. \& Bussi, G. (2016). Geographic distribution of deaths among elderly due to traffic accidents. Escola Anna Nery, 20(1), 130-137. DOI: http://dx.doi. org/10.5935/1414-8145.20160018.

Rodríguez, J. M. \& Campuzano, J. C. (2010). Medidas de prevención primaria para controlar lesiones y muertes en peatones y fomentar la seguridad vial. Revista Salud Pública, 12(3), 497-509. Recuperado de https://revistas.unal.edu.co/index.php/revsaludpublica/article/view/11214/23127.

Sadeghi, H., Samadirad, B. \& Moslemi, F. (2018). A decade of road traffic fatalities among the elderly in north-West Iran. BMC Public Health, 18(111), 1-7. Recuperado de https://www.ncbi.nlm.nih.gov/pubmed/29310628.

Segura, A. M., Cardona, D., Berbesí, D. Y. \& Agudelo, A. (2017). Mortalidad por accidente de tránsito en el adulto mayor en Colombia. Revista Saúde Pública, 51(21), 1-8. DOI: http://dx.doi.org/10.1590/s15188787.2017051006405.

Valenzuela, L. M. \& Talavera, R. (2015). Entornos de movilidad peatonal: una revisión de enfoques, factores y condicionantes. Revista latinoamericana de estudios urbano regionales, 41(123), 5-27. Doi: http:// dx.doi.org/10.4067/S0250-71612015000300001.

Vanegas, Y. \& Cárdenas, M. (2011). Años potencialmente perdidos por accidente de tránsito, Colombia 2010. Revista CES Salud Pública, 2(2), 159-168. Recuperado de file:///C:/Users/gutie/Downloads/ DialnetAnosPotencialmentePerdidosPorAccidenteDeTransitoCo-3818075.pdf.

Zangooei, H., Shafahi, Y. \& Zangooei, M. (2013). Fatal accident distribution by age, gender and head injury, and death probability at accident scene in Mashhad, Iran, 2006-2009. International Journal of Injury Control and Safety Promotion, 20(2), 121-133. DOI: https://doi.org/10.1080/17457300.2012.692694. 